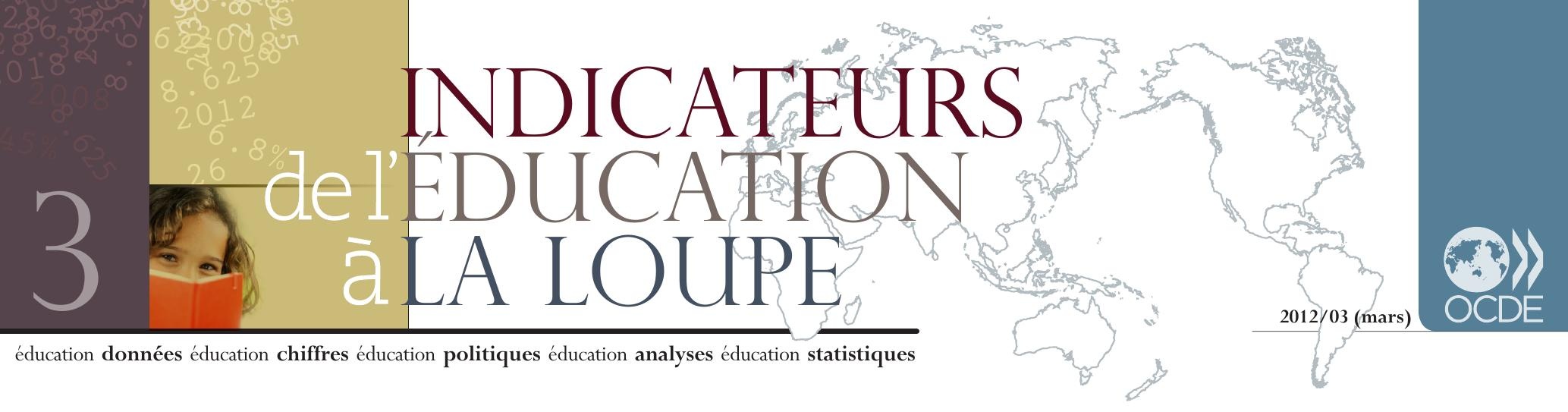

\title{
Comment les filles réussissent-elles dans leurs études - et les femmes au travail - dans le monde ?
}

- Lors de l'évaluation PISA 2009 de la performance en compréhension de l'écrit, les filles devançaient les garçons dans tous les pays de 39 points, en moyenne, soit l'équivalent d'une année d'études. Par contraste, les garçons obtenaient, en général, de meilleurs résultats que les filles en mathématiques.

- On estime que $66 \%$ des jeunes femmes des pays de I'OCDE devraient poursuivre une formation universitaire au cours de leur vie, contre $52 \%$ des jeunes hommes.

- Les hommes restent plus susceptibles que les femmes d'obtenir un titre sanctionnant un programme de recherche de haut niveau et représentent $74 \%$ de l'ensemble des diplômés dans les domaines de l'ingénierie, de la production et de la construction.

- La poursuite d'études supérieures (tertiaires) améliore les perspectives d'emploi tant pour les hommes que pour les femmes, et les inégalités entre les sexes en matière d'emploi se réduisent aux plus hauts niveaux de formation.

Dans le monde entier, les filles devancent les garçons en compréhension de l'écrit...

Alors que ce mois de mars a été l'occasion de célébrer dans le monde entier la réussite des femmes, les progrès accomplis par les filles et les jeunes femmes dans l'éducation - et par les femmes sur le marché du travail - sont incontestables. Ces dernières années, l'écart de performance entre garçons et filles à l'école s'est réduit dans des matières clés - à tel point que dans certaines d'entre elles, c'est désormais la moindre performance des garçons qui est devenue un sujet de préoccupation. À titre d'exemple, lors de l'évaluation PISA 2009 de la performance des élèves de 15 ans en compréhension de l'écrit, les filles devançaient les garçons dans tous les pays de l'OCDE de 39 points, en moyenne, soit l'équivalent d'une année d'études. En mathématiques, toujours à l'âge de 15 ans, les garçons obtenaient en général des résultats légèrement supérieurs à ceux des filles dans la plupart des pays, tandis qu'en sciences, les écarts de performance selon le sexe étaient moins prononcés.

... et les femmes représentent la majorité des nouveaux inscrits dans l'enseignement supérieur. Plus les garçons et les filles obtiennent de bons résultats à l'école, plus ils sont susceptibles de poursuivre leurs études. Entre 2000 et 2009, la probabilité pour les jeunes hommes comme pour les jeunes femmes de s'inscrire dans une formation universitaire (tertiaire de type A) a augmenté de façon spectaculaire, passant de $47 \%$ à $59 \%$. Pourtant, une fois ces pourcentages ventilés par sexe, il apparaît clairement que les femmes sont le moteur de cet essor. Ainsi, la proportion de femmes susceptibles d'entamer une formation universitaire est passée de $51 \%$ en 2000 à $66 \%$ en 2009, tandis que la proportion d'hommes susceptibles d'entamer ce type de formation est passée de $42 \%$ en 2000 à $52 \%$ en 2009.

Dans les pays de I'OCDE, la proportion de femmes diplômées à l'issue d'une formation universitaire est désormais égale, voire supérieure, à celle des hommes dans 29 des 32 pays dont les données sont comparables. En moyenne, les femmes représentent $59 \%$ de l'ensemble des étudiants diplômés à l'issue d'une première formation universitaire en 2009 ; cette proportion n'est inférieure à $50 \%$ qu'en Chine, en Corée, au Japon et en Turquie. 


\section{Pourcentage de diplômes délivrés à des femmes à l'issue d'une formation universitaire (2009)}

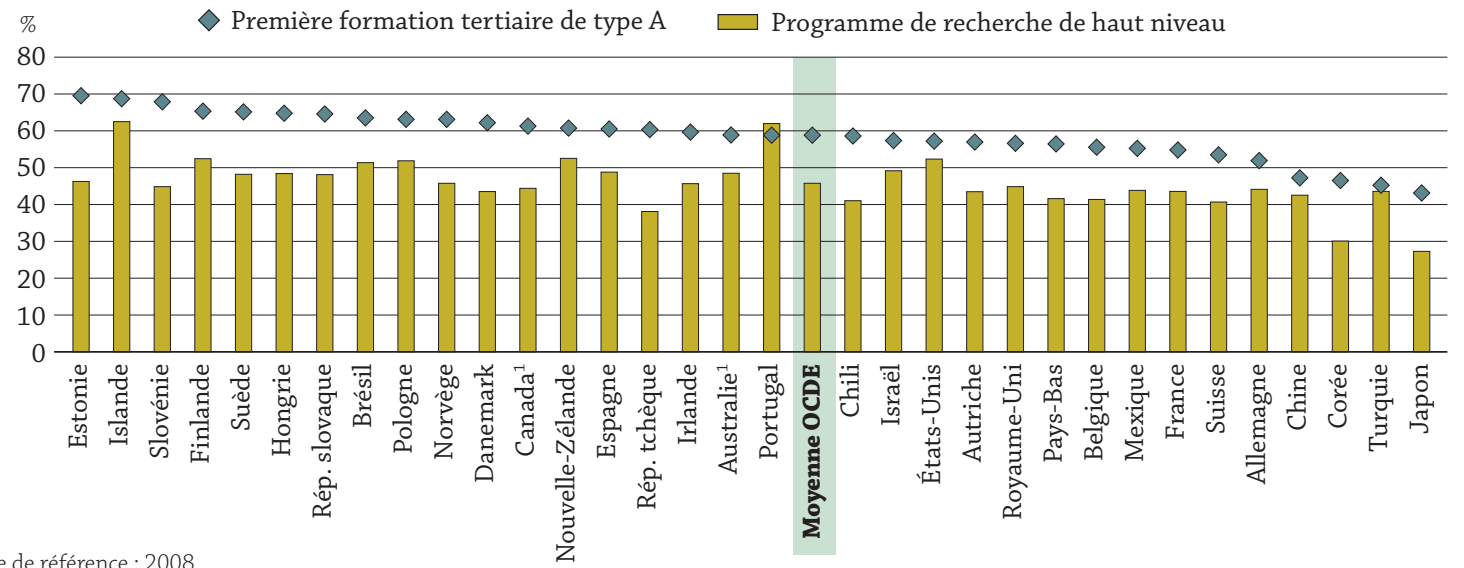

1. Année de référence : 2008 .

Les pays sont classés par ordre décroissant du pourcentage de diplômes délivrés à des femmes à l'issue d'une formation tertiaire de type A en 2009. Source : Base de données de l'OCDE sur l'éducation.

\section{Toutefois, les hommes sont plus susceptibles que les femmes d'obtenir un doctorat...}

Parallèlement, les femmes restent sous-représentées aux plus hauts niveaux de formation. En 2009, en moyenne dans les pays de l'OCDE, $54 \%$ des titres sanctionnant des programmes de recherche de haut niveau, tels que les doctorats, étaient délivrés à des hommes. Cette tendance s'observe dans l'ensemble des pays à l'exception du Brésil, de la Finlande, des États-Unis, de I'Islande, de la Nouvelle-Zélande, de la Pologne et du Portugal, où les femmes se voient désormais décerner la majorité des titres sanctionnant des programmes de recherche de haut niveau. En Corée et au Japon, seuls 30 \% environ de ces titres sont obtenus par des femmes.

... et les femmes sont sous-représentées dans certains domaines d'études.

En outre, certains domaines d'études restent considérés comme plus « masculins » et d'autres plus " féminins ». En 2009, dans chacun des pays à l'exception du Japon et de la Turquie, plus de $70 \%$ des étudiants suivant une formation universitaire dans le domaine de l'éducation étaient des femmes. En moyenne dans les pays de l'OCDE, $75 \%$ des diplômes dans le domaine de la santé et du secteur social étaient également délivrés à des femmes. Par contraste, dans la plupart des pays, les femmes représentaient moins de 30 \% de l'ensemble des diplômés dans les domaines de l'ingénierie, de la production et de la construction.

Dans quelques pays de l'OCDE, on observe actuellement un recul de l'inégalité entre les sexes dans certains domaines d'études traditionnellement considérés comme masculins. En Allemagne, en République slovaque et en République tchèque, par exemple, la proportion de femmes titulaires d'un diplôme validant des études de sciences a augmenté de plus de 10 points de pourcentage entre 2000 et 2009. Ces pays se rapprochent ainsi désormais de la moyenne de l'OCDE qui s'établit à $40 \%$ et est restée stable au cours des dix dernières années. En 2000, I'Union européenne faisait connaître son objectif d'augmenter le nombre de diplômés en mathématiques, en sciences et en technologie d'au moins $15 \%$ à l'horizon 2010, et de réduire les inégalités entre les sexes dans ces domaines d'études. À ce jour, toutefois, les progrès réalisés vers cet objectif sont restés modestes.

\section{Les progrès des femmes en termes d'éducation favorisent la réduction des inégalités} entre les sexes en matière d'emploi dans de nombreux pays...

Dans l'ensemble, cependant, les progrès importants accomplis par les femmes en termes d'éducation se traduisent par des résultats positifs sur le marché du travail. En moyenne, en 2009, 79 \% des femmes diplômées de l'enseignement supérieur avaient un emploi, contre 66 \% des femmes diplômées du deuxième cycle de l'enseignement secondaire et $46 \%$ des femmes dont le niveau de formation est inférieur au deuxième cycle de l'enseignement secondaire. 5108 . 4 


\section{Pourcentage de diplômes délivrés à des femmes à l'issue d'une formation tertiaire, selon le domaine d'études (2009)}

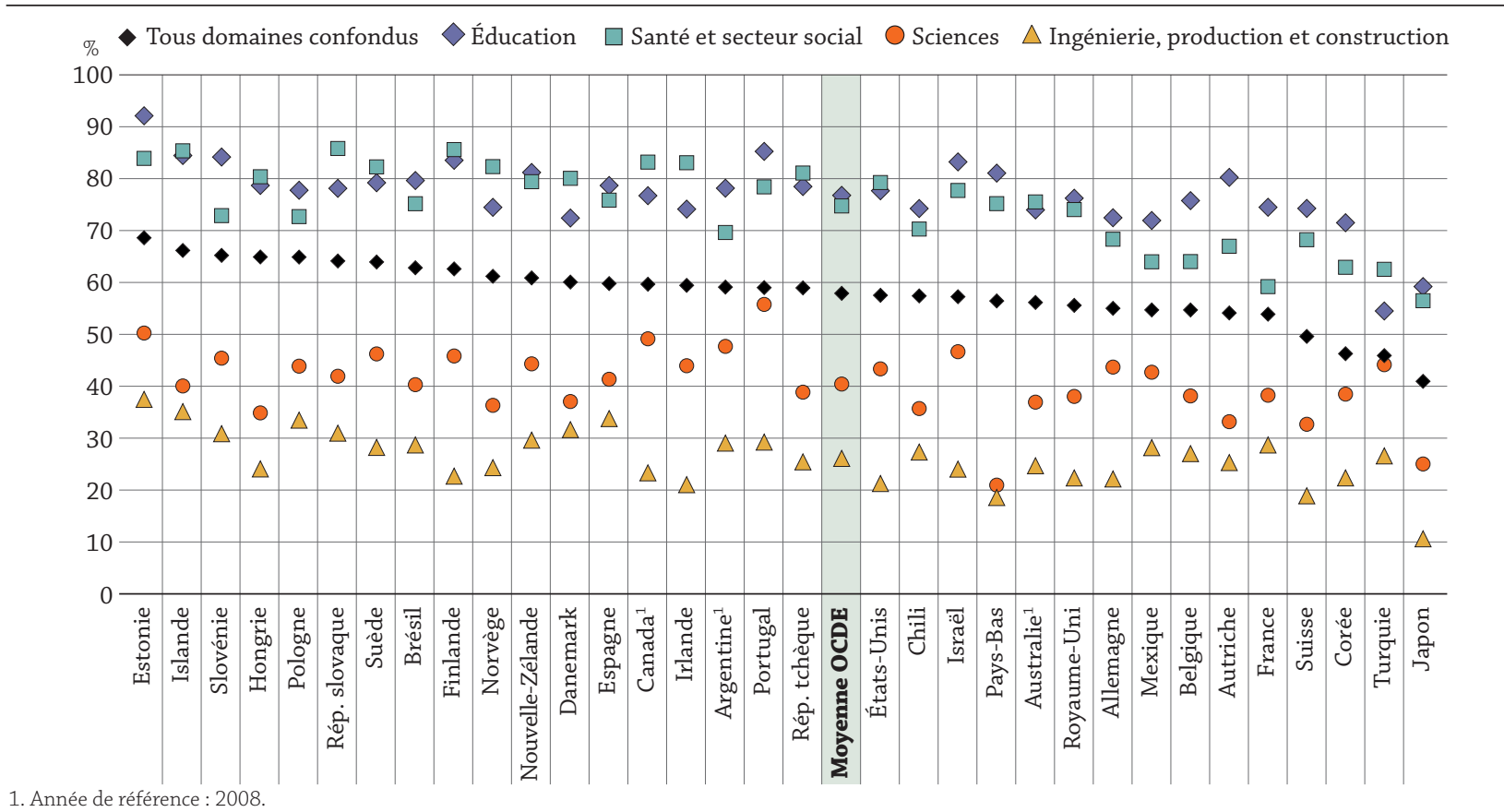

Les pays sont classés par ordre décroissant du pourcentage de diplômes délivrés à des femmes à l'issue d'une formation tertiaire en 2009.

Source : OCDE. Données relatives à l'Argentine : Institut de statistique de l'UNESCO (Programme des indicateurs de l'éducation dans le monde). Regards sur l'éducation 2011 : Les indicateurs de l'OCDE, indicateur A4 (www.oecd.org/edu/eag2011).

Il convient néanmoins de noter que les femmes sont toujours moins susceptibles que les hommes de participer au marché du travail - même à niveaux de formation identique - et sont également plus susceptibles de travailler à temps partiel.

Toutefois, au cours des dix dernières années, l'écart en faveur des hommes s'est réduit ; il est par ailleurs bien moins important aux niveaux supérieurs de formation. Ainsi, l'écart entre les sexes en matière d'emploi s'est atténué, passant de 25 points de pourcentage en 2000 à 21 points de pourcentage en 2009 parmi les individus dont le niveau de formation est inférieur au deuxième cycle de l'enseignement secondaire, et de 19 points de pourcentage en 2000 à 15 points de pourcentage en 2009 parmi les diplômés du deuxième cycle de I'enseignement secondaire. Enfin, pour les diplômés de l'enseignement supérieur, cet écart est encore plus ténu : il est passé de 11 points de pourcentage en 2000 à 9 points de pourcentage en 2009.

Ces chiffres peuvent varier fortement selon le pays : parmi les individus dont le niveau de formation est inférieur au deuxième cycle de l'enseignement secondaire, l'écart entre les sexes en matière d'emploi est ainsi inférieur à 10 points de pourcentage en Finlande, en Islande et en Norvège, mais supérieur à 40 points de pourcentage au Chili, au Mexique et en Turquie. Parmi les diplômés de l'enseignement supérieur, l'écart entre les sexes en matière d'emploi tend à se réduire sensiblement dans tous les pays de l'OCDE à l'exception de la Corée, du Japon, du Mexique et de la Turquie, où il est supérieur à 15 points de pourcentage.

... tout comme de récentes initiatives visant à promouvoir l'égalité entre les sexes en matière d'emploi. Les pays de l'OCDE s'efforcent également d'agir davantage en faveur de la réduction des inégalités entre les sexes en matière d'emploi. À titre d'exemple, dans les pays nordiques, en Allemagne et au Portugal, les pères peuvent bénéficier d'un congé parental et d'un complément de revenus pour la durée de ce congé. En Espagne, en Islande et en Norvège, le conseil d'administration de certaines sociétés doit être composé à hauteur d'au moins $40 \%$ de femmes, selon la taille de la société et le nombre de membres siégeant au conseil d'administration. 


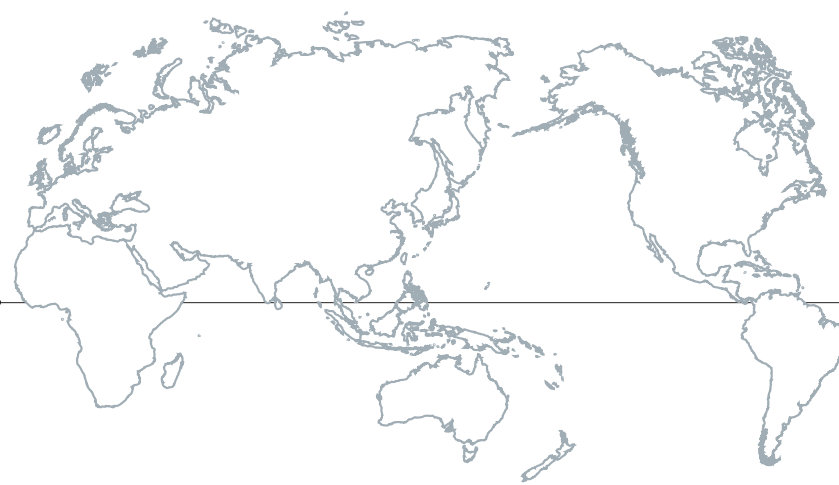

Taux d'emploi, selon le sexe et le niveau de formation (2009)

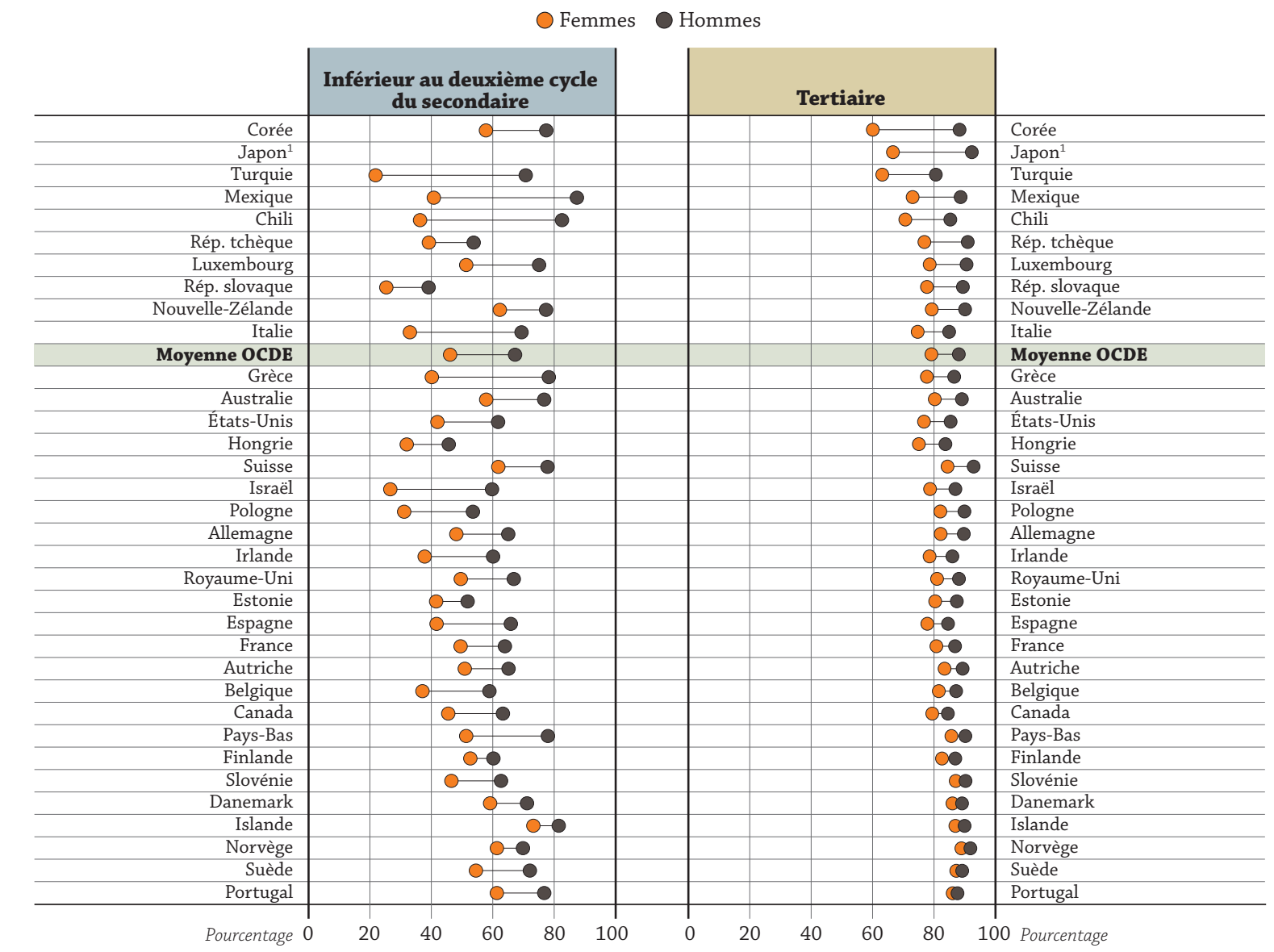

1. Les données relatives aux niveaux de formation inférieurs au deuxième cycle de l'enseignement secondaire sont manquantes.

Les pays sont classés par ordre décroissant de l'écart de taux d'emploi entre les sexes parmi les diplômés de l'enseignement tertiaire.

Source : Regards sur l'éducation 2011 : Les indicateurs de l'OCDE, indicateur A7 (www.oecd.org/edu/eag2011).

Des initiatives semblables ont été lancées en Belgique, en France, en Italie et aux Pays-Bas. Parallèlement, d'autres entreprises, telles que Deutsche Telekom, ont introduit des quotas volontaires pour la représentation des femmes aux postes de direction et des pratiques favorables à la famille telles que les horaires flexibles et le télétravail.

Toutes ces initiatives sont récentes et encore largement controversées. Elles méritent une analyse plus approfondie afin de comprendre leurs avantages en termes de débouchés pour les femmes sur le plan de l'emploi et de performance des entreprises.

Pour conclure Les initiatives visant à améliorer la performance des filles en mathématiques et en sciences - ainsi que celle des garçons en compréhension de l'écrit - peuvent promouvoir davantage encore l'égalité entre les sexes en matière d'éducation. Parallèlement, la mise en œuvre de stratégies de lutte contre les stéréotypes sexistes dans les domaines d'études et de politiques d'entreprise progressistes peut contribuer à l'amélioration des perspectives des femmes en matière d'emploi.

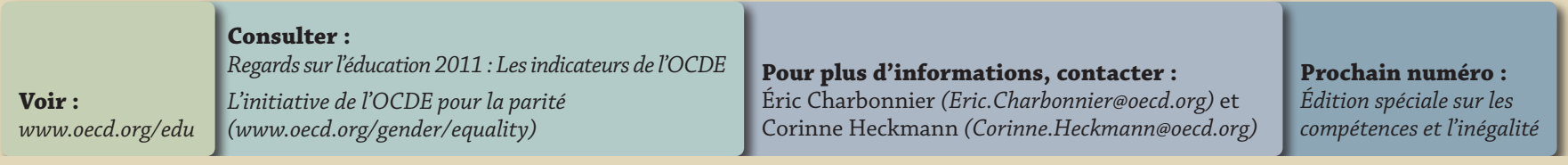

